\title{
Erratum: Critical behavior of $O(n)$-symmetric systems with reversible mode-coupling terms: Stability against detailed-balance violation [Phys. Rev. E 55, 4120 (1997)]
}

\author{
Uwe C. Täuber (1)
}

(Received 14 March 2020; published 8 May 2020)

DOI: 10.1103/PhysRevE.101.059901

In this paper, field-theoretic renormalization group (RG) methods [1] are utilized to study the universal dynamical critical scaling properties of $O(n)$-symmetric systems with reversible mode-coupling terms subject to detailed-balance violations, implemented through distinct heat bath temperatures for the critical order parameter $S$ and dynamically coupled noncritical conserved fields $M$.

Unfortunately, in the perturbative evaluation of the three-point vertex functions for the reversible mode couplings $g$ for the $O(n)$-symmetric Sásvari-Schwabl-Szépfalusy (SSS) model [2], we erroneously attached the combinatorial factors $n-1$ to the first-order fluctuation corrections [3] on the right-hand sides of Eqs. (A11) and (A12) listed in the Appendix. Interestingly, away from thermal equilibrium, this in fact invalidates the renormalization constant relation $Z_{g}=Z_{\tilde{M}}^{1 / 2}=Z_{M}^{-1 / 2}$ that follows from the Ward identity (3.17) [3]. It should also be noted that in generic nonequilibrium circumstances, the field renormalizations obtained from the two-point vertex functions are not the same as those in the corresponding dynamical response functions [4,5].

Consequently, the ensuing renormalization constants are not uniquely determined. Remarkably, there appear to exist (at least) two different consistent prescriptions to fix the resulting redundancies:

(1) One may exploit the structure of the Poisson brackets underlying the system's reversible dynamics, in symbolic shorthand $\{S, S\}=0,\{S, M\}=g S$, and $\{M, M\}=g^{\prime} M$, which imply the identities $Z_{g}=Z_{M}^{1 / 2}=Z_{g^{\prime}}[6]$ (where the distinction between the nonlinear mode couplings $g$ and $g^{\prime}$ appearing in the Langevin equations for the order parameter $S$ and the conserved quantity $M$ has been made for book-keeping purposes only).

(2) One can rescale the fields in the original Langevin equations (2.27) and (2.28) or in the Janssen-De Dominicis response functional (2.31), (2.33), and (2.35) precisely with the prescriptions (2.21) and (2.32), whereupon the noise strengths $\lambda \rightarrow \lambda$ and $\widetilde{D} \rightarrow D$ in the correlators (2.28) and (2.29), and $u \rightarrow \widetilde{u}, g \rightarrow \widetilde{g}$, and $g^{\prime} \rightarrow \widetilde{g}^{\prime}$ as defined in Eqs. (2.34) and (2.36). Formally, this rescaling procedure restores the Einstein relations between noise strengths and relaxation rates, at the price of introducing distinct mode-coupling constants; their ratio yields the nonequilibrium parameter $\Theta=\widetilde{g}^{\prime} / \widetilde{g}=\widetilde{\lambda} D g^{\prime} / \lambda \widetilde{D} g=T_{S} / T_{M}$ or effective temperature ratio for the heat baths coupled to the order parameter and conserved fields, respectively.

Naturally, procedures 1 and 2 above lead to different intermediate results for renormalization constants and RG flow functions, yet after straightforward analysis they ultimately result in the following identical RG $\beta$ functions for the couplings $w=\lambda / D$, $\Theta, f=\widetilde{g}^{2} / \lambda D=g^{2} \widetilde{D} / \lambda D^{2}=w \bar{f}$, and $\widetilde{u}$ :

$$
\begin{gathered}
\beta_{w}=w f\left[\left(\frac{1}{2}-\frac{n-1}{1+w}\right) \Theta-(n-1) \frac{w^{2}}{(1+w)^{3}}(1-\Theta)\right], \\
\beta_{\Theta}=-\frac{f}{2} \Theta(1-\Theta)\left[\Theta-\frac{1-2 w}{(1+w)^{2}}+2(n-1) \frac{1+3 w+w^{2}}{(1+w)^{3}}\right], \\
\beta_{f}=f\left[-\epsilon+\left(\frac{2-\Theta}{2}+\frac{n-1}{1+w}\right) f \Theta+\frac{w}{(1+w)^{2}}\left(2-(n-1) \frac{2+w}{1+w}\right) f(1-\Theta)\right], \\
\beta_{\widetilde{u}}=\widetilde{u}\left[-\epsilon+\frac{n+8}{6} \widetilde{u}-2(n-1) \frac{1+3 w+w^{2}}{(1+w)^{3}} f(1-\Theta)\right]+\frac{6(n-1)}{1+w} f^{2} \Theta(1-\Theta),
\end{gathered}
$$

where $d=4-\epsilon$ is the spatial dimension. Note that $\beta_{w}$ and $\beta_{\widetilde{u}}$ are as previously listed.

The modifications for $\beta_{\Theta}$ and $\beta_{f}$ do not alter the ensuing RG fixed point structure discussed, nor do they affect the stability of the equilibrium fixed point [6]. While the nonequilibrium fixed point with $\Theta^{*}=\infty$ and its properties remain unmodified as well, there are pertinent changes for the other nonequilibrium fixed point with $\Theta^{*}=0$ : While still $w^{*}=\infty$, the correct mode-coupling fixed point value is $\bar{f}^{*}=\epsilon / 2$, and consequently the $n$ dependence changes as well for $\widetilde{u}^{*}=n u_{H}^{*}=6 n \epsilon /(n+8)$. At this weak dynamic scaling fixed point, one now obtains to first order in the $\epsilon$ expansion $z_{S}=2-(n-1) \epsilon / 2<z_{M}=2$ [6].

Finally, similar corrections need to be implemented [3] for the analysis of the SSS model with spatially anisotropic nonequilibrium perturbations: There should be no factor $n-1$ in Eq. (3.14) of Ref. [4], which invalidates (3.15) and several additional intermediate results. Yet the ensuing fixed point structure and stability are not affected; only the isotropic 
nonequilibrium fixed point with $T^{*}=1 / \Theta^{*}=\infty$ needs to be altered as stated above. Correspondingly, the dynamic critical exponents at the anisotropic nonequilibrium fixed point with $T^{\|^{*}}=\infty$ and $T^{\perp^{*}}=0$ in the longitudinal sectors are $z_{S}^{\|}=$ $2-(n-1) \epsilon / 2<z_{M}^{\|}=2$, while those in the transverse sector remain $z_{S}^{\perp}=2=z_{M}^{\perp}$.

I am deeply indebted to Luca Di Carlo and Giulia Pisegna at the University of Rome Sapienza for spotting and pointing out my error to me; I am also grateful to Luca Di Carlo, Giulia Pisegna, Andrea Cavagna, Irene Giardina, and Tomas S. Grigera for subsequent very fruitful discussions.

[1] U. C. Täuber, Critical Dynamics: A Field Theory Approach to Equilibrium and Non-Equilibrium Scaling Behavior (Cambridge University Press, Cambridge, UK, 2014).

[2] L. Sasvári, F. Schwabl, and P. Szépfalusy, Physica A 81, 108 (1975).

[3] A. Cavagna, L. Di Carlo, I. Giardina, T. S. Grigera, and G. Pisegna (private communication).

[4] U. C. Täuber, J. E. Santos, and Z. Rácz, Eur. Phys. J. B 7, 309 (1999).

[5] U. C. Täuber and S. Diehl, Phys. Rev. X 4, 021010 (2014).

[6] A. Cavagna, L. Di Carlo, I. Giardina, T. S. Grigera, and G. Pisegna (private communication). 